\title{
Research and Application of Spacer Installation Robot
}

\author{
Shen Jinqing ${ }^{1}$, Yao Hongwei ${ }^{1}$, Chen Hua ${ }^{2, *}$, Zheng Shengzhong ${ }^{2}$, Lin Guiming ${ }^{2}$, Liang Wei ${ }^{2}$, Zhang Lei ${ }^{2}$ \\ 1. State Grid Zhejiang Electric Power Co., Ltd. Jiaxing Power Supply Company, Jiaxing 31400, Zhejiang Province, China \\ ${ }^{2}$.Yangtze Delta Region Institute Of Tsinghua University, Institute Of Information Technology, Jiaxing 314000, Zhejiang Province, \\ China
}

\begin{abstract}
In the current construction process of high-voltage transmission lines, the installation of spacer rods mainly adopts manual stepping on the line and high-altitude operation flying vehicles. However, the existing methods still have safety hazards and are not efficient. In the process of installing the spacer rod, it is necessary to measure and locate the installation position of the spacer rod. At present, measuring tape or ground theodolite is mainly used for measurement, which requires the cooperation of multiple people, which is inconvenient to operate. This paper proposes and designs a spacer rod installation robot, gives the basic structure and control system design of the robot, and completes the mechanical processing and assembly of the robot prototype. On this basis, the key components are debugged and tested. Finally, the prototype was tested in an experimental environment and related technical indicators were tested to verify the feasibility and effectiveness of the design scheme.
\end{abstract}

\section{Introduction}

EHV transmission technology ${ }^{[1]}$ is a cutting-edge technology that has yet to mature in the world, but its core technology, reliability and environmental impact are still to be further studied, and the construction techniques used for the installation of spacer and other accessories are also one of the significant topics of EHV research.

At present, the spacer installation ${ }^{[2][3]}$ methods are mainly: 1) construction personnel directly stepping out of line for spacer bar installation; 2) using an aerial work flyer ${ }^{[4]}$ for spacer installation; 3) aircraft-assisted installation $^{[5]}$. The above methods all have certain problems. The safety of the first method is difficult to guarantee, and the construction efficiency is low; most of the current operation flyers are not highly automated and still need a lot of manual assistance, so the efficiency is not significantly improved; the cost of aircraft-assisted installation is high.

A key technology in the installation of spacer is to carry out the measurement of spacer span distance ${ }^{[6]}$, and the current methods are mainly: 1) using tape measure or metric rope measurement; 2) using ground latitude and longitude meter measurement ${ }^{[7]}$. Both of the above methods require the cooperation of more than one person to complete, which is inconvenient to operate and has a bad impact on the improvement of construction efficiency.

The installation of spacer is dangerous and time-consuming, and many of the more dangerous tasks are gradually handed over to robots in order to ensure the safety of workers and improve efficiency. However, at the present stage, there is little research on spacer installation robots at home and abroad. Chen Sicheng et al. designed a spacer installation and maintenance robot based on the traditional transmission and substation maintenance flyer ${ }^{[8]}$ to compensate for the safety and efficiency, which can realize the automation of transmission and substation maintenance and the automation of crossing obstacles, and can automatically install spacer through system control. The authors have simulated the barrier crossing capability of the robot, completed the physical fabrication and verified the barrier crossing function in the laboratory environment. The system aims to achieve full automation of spacer bar installation, and includes how to ensure the spacer installation position, but no specific scheme and experimental verification are given.

In this paper, a spacer installation robot based on RTK positioning technology ${ }^{[9][10]}$ is designed, the basic structure and control system design of the robot are given, and the machining and assembly of the robot prototype are completed, based on which the key components are debugged and tested.

\section{Machine Structure and Operation Step Description}

\subsection{Structure Design of the Whole Machine}

A modular design scheme ${ }^{[11]}$ is adopted in the spacer installation robot proposed in this paper, as shown in Fig.1 and Fig.2, including: 1-mounted walking

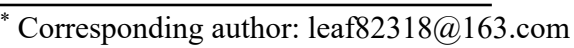


module, 2-working platform module, 3-disassembled toolbox module, 4-spacer mounting module, 5-power module, 6-visualization control module, 7-vision control module, etc. Each of the above modules can be easily disassembled and assembled, which is very suitable for carrying and using in various construction environments.

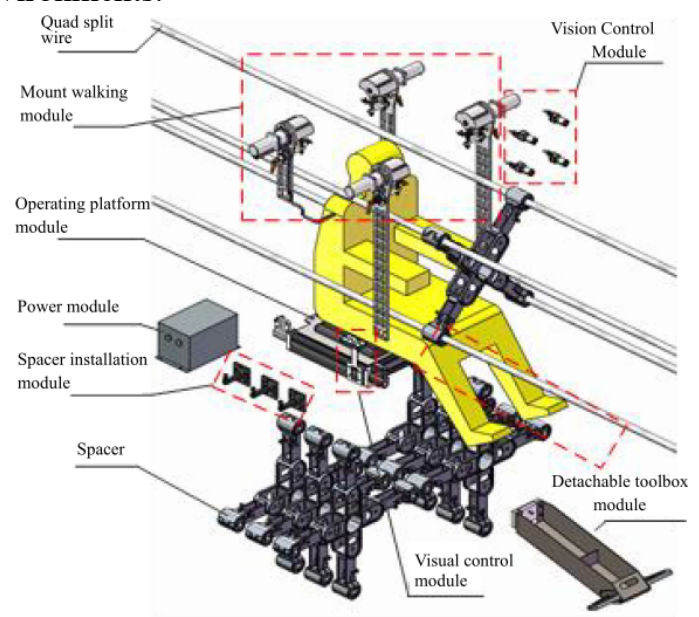

Fig. 1. Schematic diagram of the structure of the spacer installation robot (front upper 45 degree view)

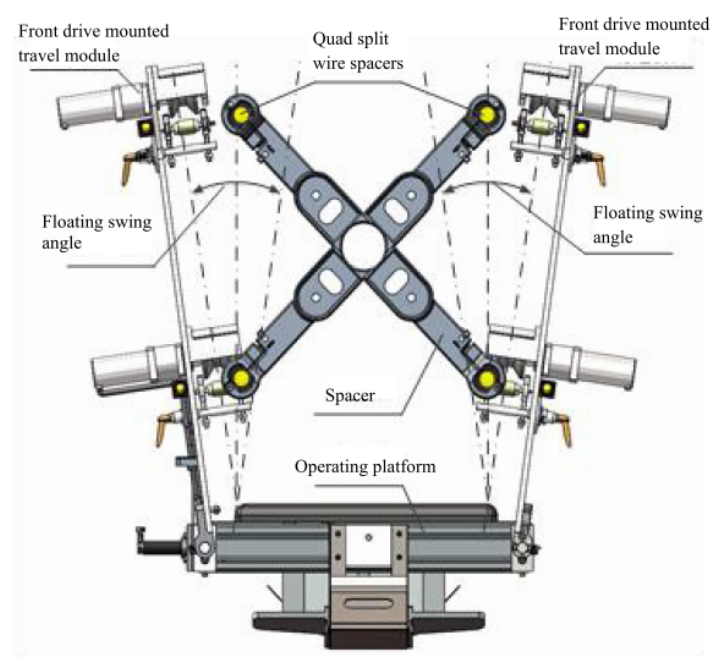

Fig. 2. Schematic diagram of the spacer installation robot structure (Rear view)

The operating platform module is the main body that connects each functional module, carrying the RTK module and the control system main board, which is connected to each functional module through each connection mechanism it sets.

The mounted travel module is mainly used to move the working platform, while the adaptive wire spacing can be adjusted, and the detachable toolbox module is used to store the working tools and pedals. The spacer mount module is used to store the spacer to be installed. The visualization control module provides the operation of forward, backward, emergency stop and reset of the working platform, and there is a visualization LCD display interface on the top of the panel, so that the construction personnel can view the running position of the equipment platform in real time, so as to install the spacer in a precise position.

The vision control module is equipped with 4 sets of industrial cameras on the front drive mounted walking module, the cameras are located near the 4 wires to detect and identify the identifiers during the installation of the spacer in real time, and determine whether the quality of the spacer installation complies with the requirements according to the location of the identified objects.

\subsection{Specific Operation Steps}

The [1-mounted walking module] is composed of two groups of front-drive mounted walking modules and two groups of rear-drive mounted walking modules, before the construction operation, first connect the [1-mounted walking module] to the [2-operating platform module] through the convenient structure, secondly put the [5-power module] into the inside of the [2-operating platform module], then connect the [3-preparable toolbox module] to the [2-operating platform module], and finally install the spacer on the [4-spacer mounting module] to complete the assembly of the spacer installation automatic work platform. After the platform is assembled, the automatic working platform is mounted to the 4-spacer wire by [1-mounted walking module], and the working platform is moved back and forth by [6-visual control module] in real time. The [7-visual control module] is located on the [1-mounted walking module], and the installation quality of the spacer is ensured by image recognition.

\section{Control System}

The control system of the spacer installation robot based on RTK positioning technology is shown in Fig. 3, including: motion control ${ }^{[12][13]}$, RTK positioning, data communication, visual recognition ${ }^{[14][15]}$, human-computer interaction interface and so on.

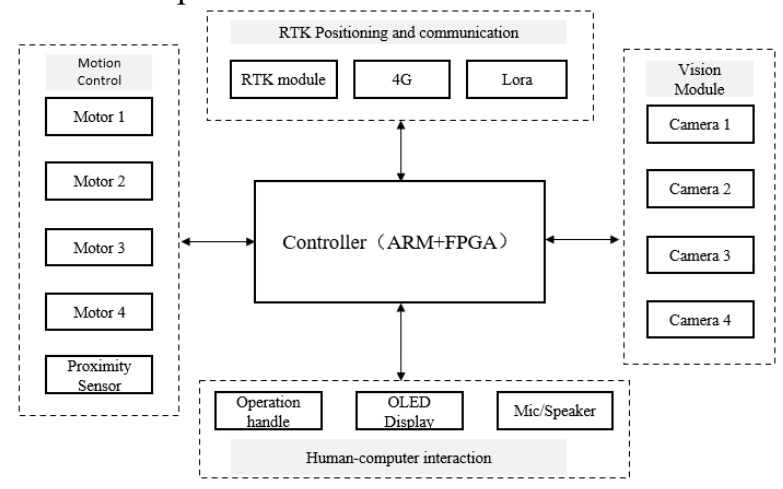

Fig. 3. Block diagram of control system

The motion control includes the four-wheel drive control of the mounted travel motor ${ }^{[16]}$, the and self-adjusting control based on the feedback from the proximity switch sensor, so that the whole platform moves according to the operation command.

The ARM circuit receives the control commands from the control handle, Lora and $4 \mathrm{G}$, and sends the commands to the FPGA after comprehensive judgment, and the FPGA generates the corresponding motor control signals to control the motor motion 
according to the commands, while collecting the motor position and current information and uploading them to the ARM circuit. The system adopts parallel control scheme for multiple motors, and adopts closed-loop control mode of speed and current loop for single motor. The speed value of the motor is obtained by T-measurement, specifically by the FPGA counting the Hall sensor of the motor in unit time, and the count divided by the time is the running speed of the motor.

RTK technology is adopted the positioning module, and the accuracy can reach centimeter level. It can realize the accurate measurement of the installation position of the spacer and prompt the installer through the man-machine interface.

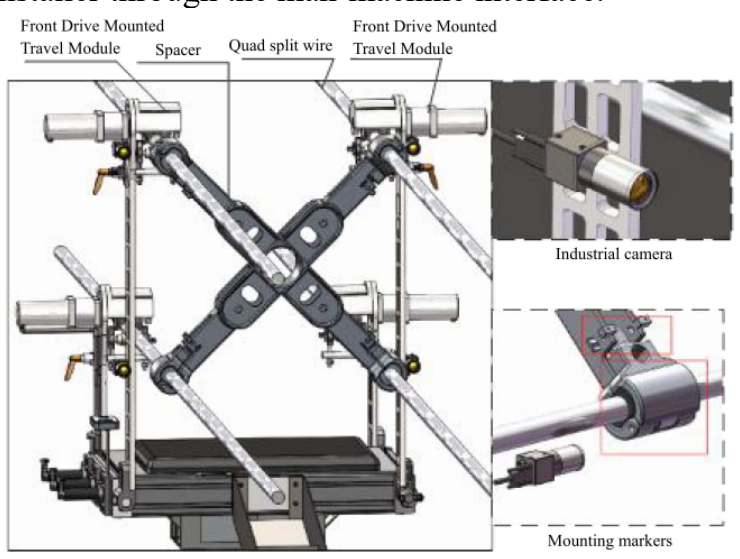

Fig. 4. Visual control

Data communication is transmitted through $4 \mathrm{G}$ or Lora, and the images during the installation process can be transmitted to the background in real time through $4 \mathrm{G}$, including the overall operating status of the robot. Lora can be used to return the robot's operating status and command control in an environment without $4 \mathrm{G}$.

Visual module mainly uses real-time image acquisition and processing analysis of 4 sets of cameras mounted on the walking module near the 4 wires to monitor the entire installation process, and finally can automatically check whether the installation quality of the spacer bar meets the requirements through vision. The human-computer interaction interface is mainly for the walking control of the working robot, seat adjustment, and installation position prompts.

\section{Experiment}

\subsection{Physical Prototype}

The actual physical prototype is shown in Fig.5:

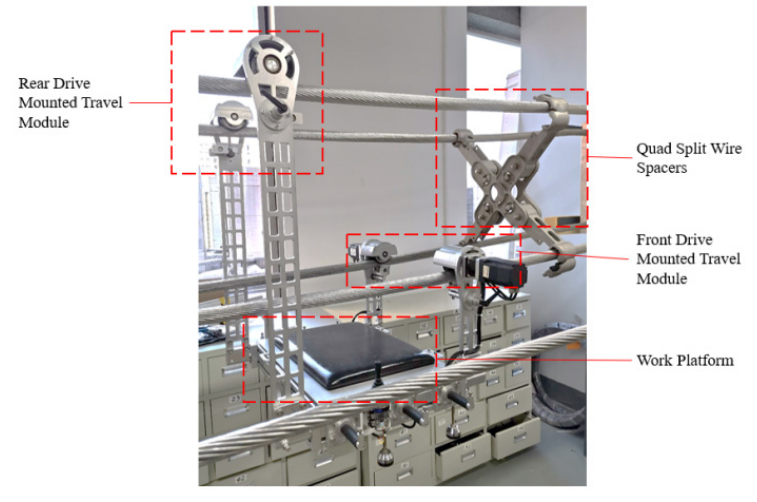

Fig. 5. Physical prototype of the robot installed with spacers

The overall performance parameters of the robot are shown in the following table:

Table 1. Performance parameter table of the robot

\begin{tabular}{lc}
\hline Robot performance & Parameter \\
\hline Total Length $\mathrm{L} / \mathrm{mm}$ & 900 \\
Total Width $\mathrm{W} / \mathrm{mm}$ & 900 \\
Total Height $\mathrm{H} / \mathrm{mm}$ & 900 \\
Weight $\quad \mathrm{M} / \mathrm{kg}$ & 60 \\
Climbing Angle /o & $\geq 25^{\circ}$ \\
Maximum Travel Speed $\quad$ Vmax & $\geq 1.2$ \\
Positioning Accuracy $/ \mathrm{cm}$ & \pm 4 \\
Continous Working Time $/ \mathrm{h}$ & $\geq 8$ \\
\hline
\end{tabular}

\subsection{RTK Positioning Accuracy Test Method}

1. Set 5 locations at a fixed height, numbered from 1 to 5. Move the robot equipped with RTK module to these 5 positions in turn, and record the positioning data;

2. Use the positioning data recorded by RTK to calculate the distance between the position points and compare with the value measured by the tape measure.

\subsection{RTK Positioning Accuracy Test Results}

Table 2. RTK return data table of the position to be

\begin{tabular}{|c|c|c|c|c|c|}
\hline \multicolumn{6}{|c|}{ measured } \\
\hline Location Label & 1 & 2 & 3 & 4 & 5 \\
\hline Longitude & $\begin{array}{c}120^{\circ} \\
49^{\prime} 18.44152^{\prime \prime}\end{array}$ & $\begin{array}{c}120^{\circ} \\
49^{\prime} 18.44100^{\prime \prime}\end{array}$ & $\begin{array}{c}120^{\circ} \\
49^{\prime} 18.42354^{\prime \prime}\end{array}$ & $\begin{array}{c}120^{\circ} \\
49^{\prime} 18.46005^{\prime \prime}\end{array}$ & $\begin{array}{c}120^{\circ} \\
49^{\prime} 18.40135^{\prime \prime}\end{array}$ \\
\hline Latitude & $30^{\circ} 44^{\prime} 16.07661^{\prime \prime}$ & $30^{\circ} 44^{\prime} 16.08520^{\prime \prime}$ & $30^{\circ} 44^{\prime} 16.08727^{\prime \prime}$ & $30^{\circ} 44^{\prime} 16.07288$ & $30^{\circ} 44^{\prime} 15.98412$ \\
\hline Elevation(m) & 29.176 & 29.195 & 29.192 & 29.18 & 29.195 \\
\hline HRMS(m) & 0.01 & 0.008 & 0.006 & 0.019 & 0.008 \\
\hline VRMS(m) & 0.013 & 0.01 & 0.008 & 0.018 & 0.011 \\
\hline $\begin{array}{l}\text { Longitude of } \\
\text { reference station }\end{array}$ & $\begin{array}{c}113^{\circ} \\
21^{\prime} 59.82440^{\prime \prime}\end{array}$ & $\begin{array}{c}113^{\circ} \\
21^{\prime} 59.82440^{\prime \prime}\end{array}$ & $\begin{array}{c}113^{\circ} \\
211^{\prime} 59.82440^{\prime \prime}\end{array}$ & $\begin{array}{c}113^{\circ} \\
2159.82440^{\prime \prime}\end{array}$ & $\begin{array}{c}113^{\circ} \\
211^{\prime} 59.82440 "\end{array}$ \\
\hline $\begin{array}{c}\text { Latitude of } \\
\text { reference station }\end{array}$ & $23^{\circ} 7^{\prime} 35.67690^{\prime \prime}$ & $23^{\circ} 7^{\prime} 35.67690^{\prime \prime}$ & $23^{\circ} 7^{\prime} 35.67690^{\prime \prime}$ & $23^{\circ} 7^{\prime} 35.67690^{\prime \prime}$ & $23^{\circ} 7^{\prime} 35.67690^{\prime \prime}$ \\
\hline $\begin{array}{c}\text { Elevation of } \\
\text { reference }\end{array}$ & 30 & 30 & 30 & 30 & 30 \\
\hline
\end{tabular}


Table 3: RTK measurement error table

\begin{tabular}{|c|c|c|c|}
\hline Item & $\begin{array}{l}\text { Distance } \\
\text { measured } \\
\text { by tape } \\
\text { measure } \\
(\mathrm{cm})\end{array}$ & $\begin{array}{c}\text { Distance } \\
\text { calculated } \\
\text { from latitude } \\
\text { and longitude } \\
(\mathrm{cm})\end{array}$ & $\begin{array}{l}\text { Differenc } \\
\mathrm{e}(\mathrm{cm})\end{array}$ \\
\hline Position 1-2 & 24.5 & 26.85 & -2.35 \\
\hline Position 1-3 & 55.6 & 57.74 & -2.14 \\
\hline Position 2-3 & 47.5 & 46.5 & 1 \\
\hline Position 1-4 & 54 & 50.2 & 3.8 \\
\hline Position 1-5 & 304 & 305 & -1 \\
\hline
\end{tabular}

The data values returned by RTK are shown in Table 2. According to the comparison between the returned data and the actual measurement distance, the measurement error can be obtained, as shown in Table 3 . It can be seen from the experimental results that directly from the HRMS (horizontal accuracy, unit: $m$ ) and VRMS (elevation accuracy, unit: $\mathrm{m}$ ) output by the RTK device, the horizontal accuracy is within $1 \mathrm{~cm}$ and the elevation accuracy is within $2 \mathrm{~cm}$. The error between the distance measured by the tape measure and the distance calculated according to the latitude and longitude is within $+/-4 \mathrm{~cm}$.

\section{Conclusion}

Aiming at the environmental characteristics of the installation of spacer in power transmission and transformation lines, and drawing on the advantages and disadvantages of existing institutions in the market, this paper proposes a new type of spacer installation robot, which can adapt to different construction environments and improve the installation of spacer s. Safety and efficiency, the installation accuracy of the spacer meets the requirements, and the expected effect is achieved.

\section{Acknowledgments}

State Grid Zhejiang Electric Power Company Science and Technology Project: Research on key technologies of spacer installation and maintenance robots (5211JX1900CU)

\section{References}

1. Liu, Z.Q. (2020) Research and application of key technology of AC UHV transmission line. Electron. Technol. Software Eng., 14: 228-229.

2. Luo, X.M. (2012) Analysis on the installation design of spacer rods in high-voltage overhead lines. Power Supply Tech. Appl., 10: 41-42.
3. Yang, S.Y., Su, Y.H., Chen, B.P. (2019) Design scheme of spacer rod manual, mechanical power general disassembly tool. China Metal Bulletin, 12: 112-113.

4. Xu, S.Q., Liu, K. (2009) Development of UHV Electric Folding Speed Vehicle. Electric Power Constr., 30: 90-93.

5. Ouyang T.T., Ye, L., Chen, J.P. (2011) The first live operation of a helicopter in high-altitude areas in China. Yunnan Electric Power, 4: 51-54.

6. Gou, J.H, Wei, X.D. (2018) Application of spacer positioner in modern UH voltage transmission line engineering. Sci. Inform. Tech., 3: 102-105.

7. Li, H.J. (2000) The method of controlling the installation distance of spacer rods with theodolite. Yunnan Electric Power Tech., 3:50.

8. Chen, S.C., Yan, W.Y., Guo, H.M., etc. (2019) Development of a robot for installation and maintenance of power transmission and transformation spacer bars. Transm. Distrib. Eng. Tech., 8: 81-88.

9. Yuan, Y.B., Mi, X.L., Zhang, B.C. (2020) Initial assessment of single- and dual-frequency BDS-3 RTK positioning. Satel. Navig., 1: 31.

10. Qi, E.J., Peng, D.G., Guan, X.L., etc. (2018) Research on patrol robot nabigation system based on RTK Beidou and laser radar. Inst. Tech. Sensor, 6: 58-63.

11. Feng, H.J., Miao, Q., Fan, S.Q., etc. (2019) Modular design for mechanical structure of industrial robots. Mech. Eng. Autom., 2: 100-101, 104.

12. Zhu, H.B., Li, Y. (2019) Realization of incremental PID differential car tracking based on RTK. Modern Comput., 36: 8-11

13. Xie, D.S., Xu, Y.C., Wan, J., etc. (2017) Trajectory tracking control of wheeled mobile robots based on RTK-GPS. Robot, 2: 221-229.

14. Xiong, Y.L., Luo, Z.Y., Guo, B.M. (2020) Binocular vision positioning in UAV power inspection. Integrated Circuit Appl., 4: 94-95.

15. An, Z.D., Huang, F. (2018) Real-time positioning and recognition of key components of transmission tower lines under drone vision. Artif. Intellig. Robot Res., 4: 171 -177.

16. He, Z.Y., Wang, W.G., Huang, L.(2020) Research on coordinated control of straight driving stability of Four-Wheel independent drive electric vehicle. Mach. Design Manufact., 5: 39-43. 